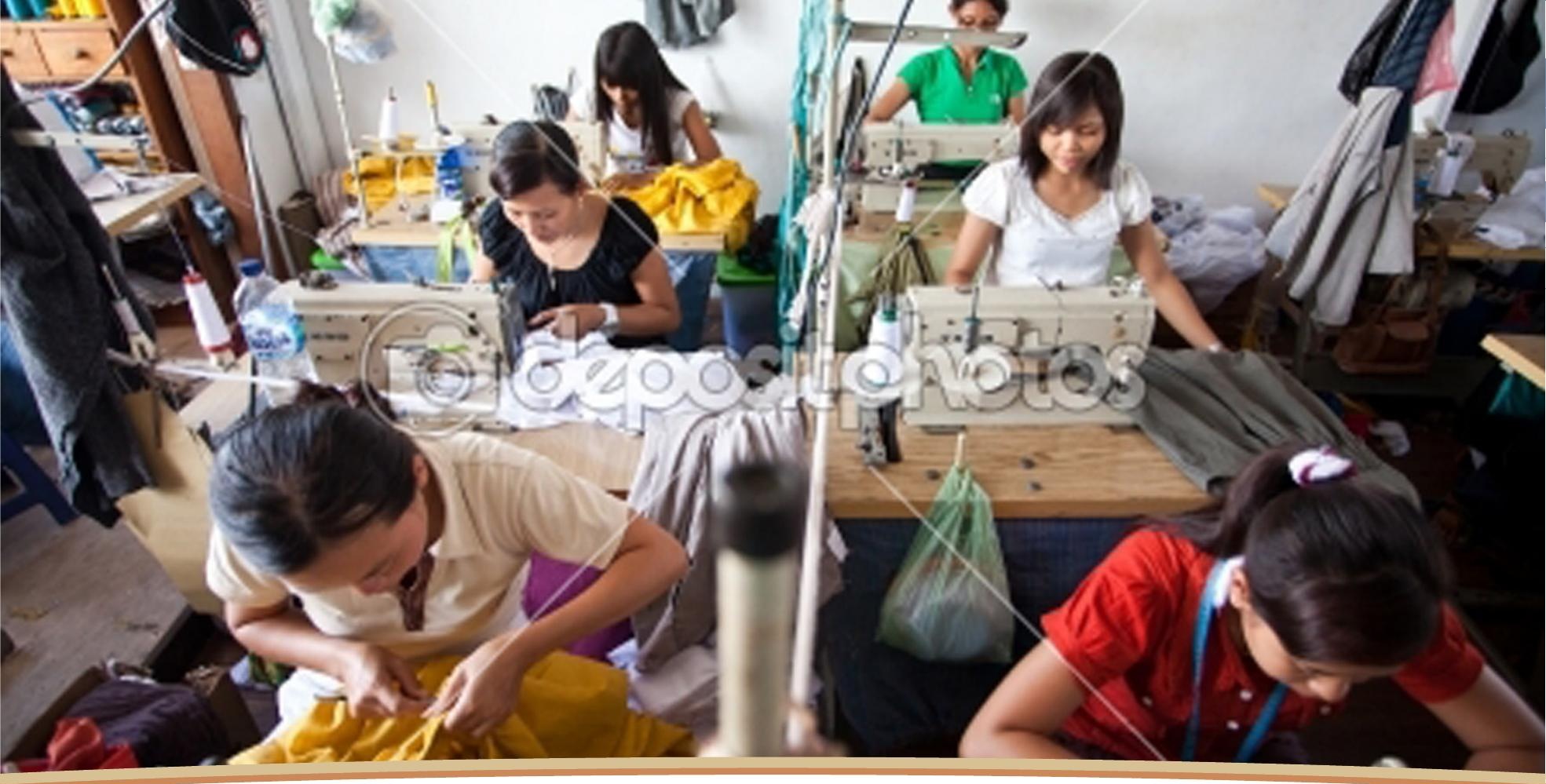

EIDOS, 5

Marzo - Agosto 2012, 35-39

ISSN:1390-5007

\title{
MODELO PARA GENERAR VALOR ECONÓMICO AGREGADO, A LAS PYMES DEL SECTOR MANUFACTURERO DE LA CIUDAD DE QUITO
}

J. Rosado', F. Coral², Universidad Tecnológica Equinoccial

Recepción/Received: 2011-11-28

Aceptación/Accepted: 2012-05-12

Publicado/Published: 2012-08-20

Resumen - El presente artículo propone un modelo orientado a mejorar la rentabilidad económica de las pymes del sector manufacturero de la ciudad de Quito. En esta investigación se ha considerado a las decisiones de operación, inversión, financiamiento, y a las políticas de motivación a los empleados como las estrategias que permiten generar valor económico agregado.

El propósito del modelo es contribuir al desarrollo sostenido de la pequeña y mediana empresa. Consciente de que se requiere un urgente cambio en los modelos de dirección para sobrevivir en un mercado globalizado y de alta competitividad

Los resultados obtenidos de la investigación permitieron seleccionar las estrategias que permitirán generar valor económico agregado como son: reducir tiempos muertos, eliminar errores de precios, invertir en propaganda y publicidad, satisfacción del cliente, optimizar el portafolio de producto, reducir los costos fijos, innovación de producto, investigación de nuevos mercados, reducir el tiempo de ciclo de productos, adquisición de tecnología de punta, invertir en imagen corporativa, invertir en sistema de gestión de calidad, reducción de las cuentas por cobrar, identificar problemas de cobranzas, vender activos ociosos, mejor la coordinación con los proveedores, obtener créditos en la bolsa de valores o corporación financiera nacional, participación de los empleados en la toma de decisiones, crear un buen clima laboral, cultura permanente de capacitación, programas de salud ocupacional.

Palabras clave - decisiones financieras, valor económico agregado.

\begin{abstract}
This article proposes a model aimed at improving the profitability of SMEs in the manufacturing sector of the city of Quito. This research has considered the decisions of operating, investing, financing, policy and employee motivation and strategies for generating economic value added. The purpose of the model is to contribute to sustainable development of small and medium enterprises. Aware that requires an urgent change in management models to survive in a globalized and highly competitive. The results of the investigation allowed to select the strategies that will generate economic value added such as: reducing dead time, eliminate pricing errors, reverse propaganda and advertising, customer satisfaction, optimize product portfolio, reducing fixed costs, innovation product, researching new markets, reduce product cycle time, acquisition of technology, investing in corporate, investment in quality management system, reduction of accounts receivable collection problems identified, sell idle assets, better coordination with suppliers, obtain credit in the stock exchange or national financial corporation, employee participation in decision-making, create a good working environment, culture, ongoing training, occupational health programs.
\end{abstract}

Keywords - financial decisions, economic value added

1 Jhon Rosado Varela, rvjf44483@ute.edu.ec, Universidad Tecnológica Equinoccial

${ }^{2}$ Fernando Coral, fcoral@ute.edu.ec, Docente de Posgrados, Universidad Tecnológica Equinoccial 


\section{INTRODUCCIÓN}

Uno de los objetivos de la empresa con fines de lucro es la de generar ganancias que cubran las expectativas de los accionistas, inversionistas, empleados y sociedad. En este contexto la responsabilidad del gerente o administrador es la de identificar las estrategias que generan valor. Las inversiones, nuevos mercados, innovación, tecnología, el desarrollo del talento humano, forma parte de la agenda de las grandes empresas ecuatorianas; pero en contraste a esta realidad, las Pymes ecuatorianas siguen presentando problemas como: la carencia de un plan estratégico, un modelo de gestión de calidad, y herramientas de control, que por el contrario reducen su capacidad de expandirse en los mercados internos como externos.

Las decisiones que se tomen en las pequeñas y medianas empresas es un proceso clave para mejorar la rentabilidad económica, las decisiones correctas conducen a crear valor para los accionistas, inversionistas, empleados y sociedad, por el contrario las decisiones equivocadas destruyen valor.

Por esta razón, el proyecto tiene como objetivo proponer un modelo que permita a las pequeñas y medianas empresas orientar generación de valor económico agregado, a través de las decisiones de inversión, de operación, de financiamiento, y la aplicación de políticas de motivación a los empleados, como base de la presente investigación.

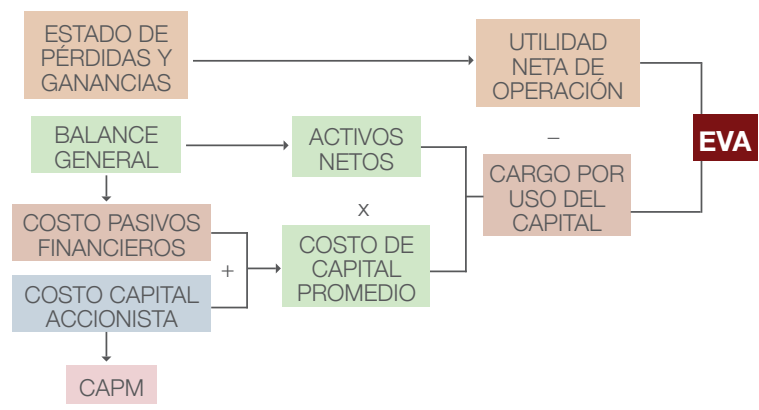

Figura 1. Estructura General del EVA

Fuente: Tomado del Grupo Internacional de Asesoría (2011)

\section{LA ESTRATEGIA DE OPERACIÓN}

La estrategia de operación es individual, propia de cada empresa, pero cabe enunciar ciertas tendencias
MODELO PARA GENERAR VALOR ECONÓMICO AGREGADO...

generales, según la época y el país. En una perspectiva histórica por ejemplo, en los EE.UU en los años posteriores a la Segunda Guerra Mundial, las empresas experimentaron una fuerte demanda de parte de sus clientes, demanda que había quedado insatisfecha durante la guerra, por lo cual orientaron sus estrategias de operaciones hacia la producción masiva de artículos estandarizados. Durante el mismo período, Japón, en su necesidad de reconquistar mercados exteriores, orientó su estrategia de operación hacia la optimización de la relación entre calidad y costo.

Las Pymes deben entender que para competir en los mercados actuales se hace necesario redefinir los procesos operativos, tomando en cuenta que las necesidades de los clientes cambian constantemente. El modelo propuesto hace referencia a las principales decisiones operativas como es reducir tiempos muertos, eliminar errores de precio y de pedido, invertir en propaganda publicidad, mejorar la satisfacción del cliente, optimizar el portafolio de productos y reducir los costos fijos.

\section{LA ESTRATEGIA DE INVERSIÓN}

La decisión de invertir en una empresa requiere de un análisis que permita delinear los objetivos de la empresa, con el portafolio de inversiones, tomando como referencia las siguientes estrategias:

1. Invertir incluso más que lo que genera el producto con el fin de "comprar" participación de mercado y lograr altos niveles de rentabilidad en el futuro.

2. Invertir en el producto lo necesario para mantener su participación de mercado, generando así un flujo de rentabilidad estable a lo largo de los años.

3. Sacrificar el futuro del producto y decidir obtener el máximo de beneficio a corto plazo.

Las tres estrategias son válidas, todo depende del mercado, su estructura y las aspiraciones de las empresas.

La decisión de invertir no es común en las Pymes, pero es necesario tomar en cuenta que la innovación de nuevos productos, investigación de nuevos mercados, adquisición de tecnología de punta, invertir en la imagen corporativa, invertir en gestión de calidad deben formar parte de toda empresa que quiera crecer a corto y largo plazo. 


\section{ESTRATEGIA DE FINANCIAMIENTO}

La estrategia financiera de las empresas incluye una visión a corto y largo plazo, tomando en cuenta lo siguiente:

- Sobre el capital de trabajo

- Sobre el financiamiento corriente

- Sobre la gestión del efectivo

- Sobre la inversión.

- Sobre la estructura financiera.

- Sobre la retención y/o reparto de utilidades.

La decisión de financiamiento de las Pymes ecuatorianas que propone este modelo debe emplearse de acuerdo a las características individuales de cada empresa, entre las cuales tenemos: reducir las cuentas por cobrar, vender activos ociosos, estructurar políticas de financiamiento a corto y largo plazo.

\section{POLÍTICAS DE INCENTIVOS Y MOTIVACIÓN A LOS EMPLEADOS}

Dependiendo de sus recursos económicos, las pequeñas y medianas empresas idean estrategias para mantener a sus empleados en un buen ambiente laboral que los lleve a brindar lo mejor de sí y a ser cada vez más productivos.

Las pequeñas empresas, o aquellas que cuentan con recursos limitados, requieren de estrategias de motivación laboral que en el nivel macro brinde a los empleados un espacio propicio donde puedan desarrollarse de manera personal y profesional.

El modelo recomienda, según la realidad de cada Pyme, utilizar las siguientes estrategias: bonos sobre la producción, cultura permanente de capacitación, programa de salud ocupacional, participación en la toma de decisiones.

\section{ESTRATEGIA DE FINANCIAMIENTO}

Los pasos para realizar la investigación fueron:

- Revisión bibliográfica del valor económico agregado Pymes, política de incentivos a empleados, se lo realizó en dos fases la primera a través del internet y la segunda visitas a las bibliotecas más importantes de Quito.

- Visita a la Superintendencia de Compañía, a la Sección Dirección de Estudios Económicos Societario, para obtener información objeto de estudio.

- Visita a las Pymes seleccionadas dentro del ámbito de la manufactura, según los resultados de la muestra.

- Elaboración y aplicación de los instrumentos de recolección de datos aplicados a los gerentes y administradores de las empresas, en este caso utilizaremos el cuestionario con preguntas dicotómicas y de selección múltiples.

- Tabular, organizar y clasificar la información, a través del programa SPSS versión 17.

- Elaboración de gráficos que permitan la interpretación y análisis de los datos.

- Elaboración de las conclusiones y recomendaciones como resultado final de la investigación.

- Determinación de las variables que permitan crear un Modelo de valor económico agregado para Pymes de la ciudad de Quito.

\section{POBLACIÓN}

La población objeto de estudio, estuvo comprendida por 404 Pymes que pertenecen al sector manufacturero de la ciudad de Quito, esta información fue proporcionada por la Superintendencia de Compañías.

INSTRUMENTOS DE

RECOLECCIÓN, Y ANÁLISIS DE LOS DATOS

Se utilizó un cuestionario dividido en cuatro secciones para analizar las variables objeto de estudio, información que fue procesado en el paquete estadístico SPSS versión 17, que permitieron seleccionar las variables que permiten generar valor económico agregado.

\section{HIPÓTESIS}

La hipótesis planteada en el proyecto es:

"El modelo de valor económico agregado orientará en la creación de valor a las Pymes del sector de la manufactura de la ciudad de Quito". 
ESPECIFICACIÓN DEL MODELO

PROPUESTO

Para reconocer como las estrategias de operación, inversión, financiamientos y las políticas de incentivos y motivación a los empleados generan valor económico agregado (EVA) se elaboró el siguiente esquema:

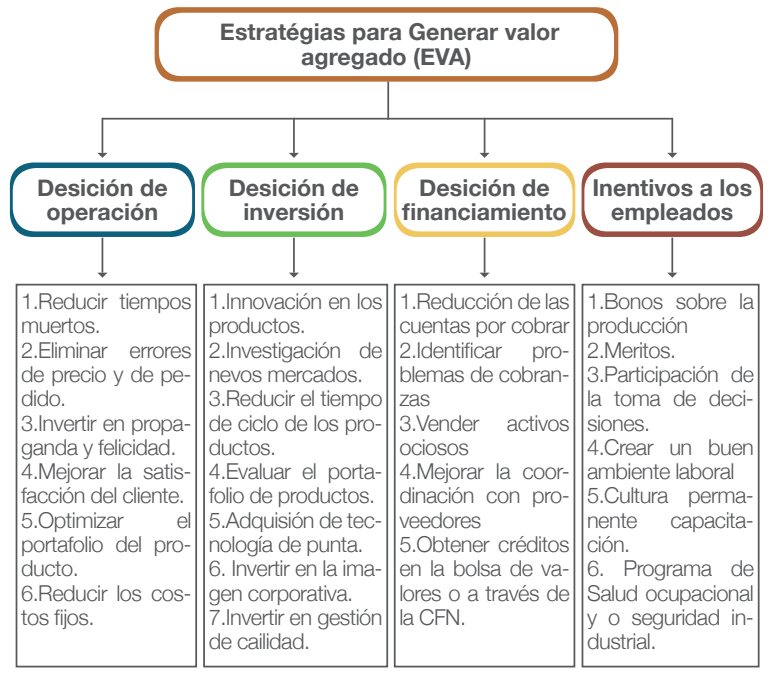

Figura 2. Modelo para generar valor económico agregado.

Elaboración: Autor

La aplicación práctica de las estrategias del presente modelo dependerá de la naturaleza de cada Pyme, como se mencionó anteriormente, cada organización debe determinar a través de un análisis integral que estrategias se adaptan a la organización. El gerente o administrador puede identificar y revaluarlos de modo que se pueden mejorar las acciones que proporcionan valor. Las decisiones de la gerencia producirán un efecto positivo cuando sean aplicadas correctamente.

\section{CONCLUSIONES}

- El modelo propuesto identifica directamente al talento humano no como un medio más para lograr la maximización de las utilidades, sino como el medio más importante para crear valor, en la organización.

- El Modelo sugiere una estrecha relación entre las decisiones de operación, inversión, financiamiento, y las políticas de motivación a los empleados como las principales fuentes, para generar valor económico agregado a las Pymes.

- El éxito del modelo propuesto dependerá de las si-
MODELO PARA GENERAR VALOR ECONÓMICO AGREGADO...

guientes condiciones:

- implementación de un plan de incentivos basado en el EVA.

- La convicción de la gerencia de liderar el programa, conjuntamente con un buen talento humano.

- La aplicación del EVA, es un cambio radical, con relación al método tradicional de medición, ya que requiere una presión constante por parte de los dueños de las empresas.

- Cultura empresarial, optimista para implantar el EVA.

\section{BIBLIOGRAFÍA}

[1] Acosta, G. (2009). El Valor Económico Agregado. EIDOS, 24-25-26.

[2] Amat, O. (2002). Eva un enfoque para optimizar la gestión, motivar y crear valor. Barcelona España: Norma (2002).

[3] Arnoletto, E. (s.f.). Biblioteca virtual de derecho, economía, y ciencias sociales. Recuperado el 06 de abril de 2012, de http://www.eumed.net/ libros/2007c/333/estrategia\%20de\%20operaciones.htm

[4] Emprendepyme. (s.f.). Recuperado el 06 de abril de 2012, de http://www.emprendepyme.net/cual-es-laestrategia-de-inversion-y-rentabilidad.html

[5] Escalera, C., \& Santiago, H. (2009). Modelo para generar valor económico agregado. Contribuciones a la economía, 21.

[6] Reyes, E., \& Díaz, K. (s.f.). Gestiopolis.com. Recuperado el 06 de abril de 2012, de http://www. gestiopolis.com/finanzas-contaduria/principalesestrategias-financieras-de-las-empresas.htm

[7] Rosado, J, \& Coral, F. (2011). Modelo para Generar Valor Económico Agregado en las Pymes del Sector Manufacturero de la ciudad de Quito. Quito: UTE.

[8] Stewart, B. (1990). The Quest for Value. USA: Harper Business. 


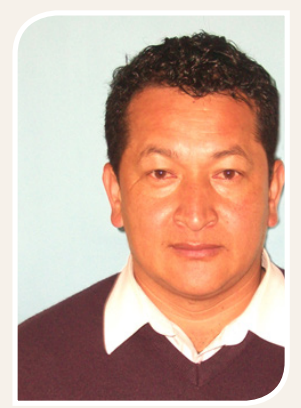

Obtuvo su Maestría en Auditoría y Finanzas en la Universidad Tecnológica Equinoccial, en el año 2012. Se graduó de Licenciado en Ciencia de la Educación en la Especialidad en Contabilidad y Administración.

Ha sido docente de varias Instituciones educativas de nivel secundario y Superior. Asesor de instituciones públicas en el manejo de la gestión administrativa y financiera.

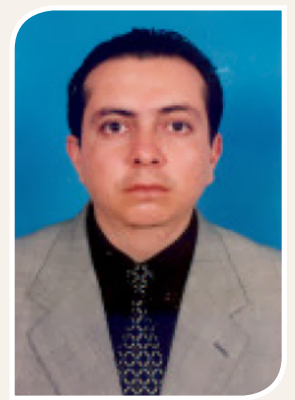

Fernando Coral

Obtuvo su Maestría en Administración de Empresas con Mención en Negocios internacionales (MBA) en la Pontificia Universidad Católica del Ecuador, en el año 2006. Se graduó de Licenciado en contabilidad y Auditoría Contador Público Auditor (CPA) en el año 2000. Obtuvo el Doctorado en Contabilidad y Auditoría en la Universidad Central del Ecuador año 2000.

Es docente de Posgrados en la a Universidad Tecnológica Equinoccial.

Ha trabajado en varias empresas del Sector Privado como Contador, Auditor, consultor. 